\title{
Physical Description and Analysis of Doped Carbon Nanotube Interconnects
}

\author{
Jie Liang, Liuyang Zhang, Nadine Azemard-Crestani, Pascal Nouet, Aida Todri-Sanial \\ CNRS-LIRMM/University of Montpellier \\ Montpellier, France \\ Email: aida.todri@lirmm.fr
}

\begin{abstract}
Ever since the discovery of graphene, the 2D carbon structure material has been attracting a lot interest due its electrical, thermal and mechanical properties. Here, we investigate the carbon nanotubes (CNT), wrapping a 2D graphene sheet to form a 1D carbon structure. CNT has a Diraccone energy band, which makes it either a semiconductor or metal. With continuous aggressive scaling, the technology using copper as interconnect is reaching its limit on current density, thermal and conductivity performance, thus finding a new interconnect material is an on-going quest. CNT with its high conductivity, high electro-migration, and low temperature effect opens a door for on-chip interconnect applications. However, the uncontrollable chirality of pure CNT motivates us to explore doped CNTs. Doping alleviates the need to control chirality and enhances the metallic property of CNTs. In this work, we start first to describe the fundamental physics of doped CNTs and describe how doping changes the electrical property of CNTs. We also provide analytical models and simulation results on CNT conductance variation for N-type doped CNTs. Simulation results show that doping can reduce overall CNT resistance by $89 \%$.
\end{abstract}

Keywords-Carbon nanotube interconnects, Doped CNT; onchip interconnect; electrical properties of doped CNT

\section{INTRODUCTION}

T here is a lot of interest and on-going research on graphene and carbon nanotubes as a viable material for faster and energy efficient devices, next generation of organic solar cells, and the material for fast-charging batteries. Research on graphene dates as early as 1946 , by Canadian physicist Philip Russel Wallace with his theoretical insights while doing research on graphite [1]. In 1991, Iijima first observed the existence of carbon nanotube during his study on synthesis of fullerenes [2]. The interest on graphene grew larger in 2004 when physicists Andre Geim and Konstantin Novoselov won the Nobel Prize of Physics by the discovery of graphene using "scotch tape method" [3-4]. This discovery made strong waves in scientific community with lots of scientist investigating how the physical properties of graphene changed when electrons are confined and forced to travel in a 2D material. Carbon nanotubes, a 1D carbon material also became of interest for their electrical, thermal and mechanical properties and particularly for nanoelectronics applications.

Carbon nanotubes are currently being investigated as potential replacement of back-end-of-line interconnect copper material for integrated circuits. The limit of conventional copper interconnect has motivated a lot of research to find another high conductive and energy efficient material. Carbon nanotubes have a semiconducting or metallic property due to their special Dirac energy band structure and open a field for nano- or micro-electronic devices and interconnect applications. However, its uncontrollable chirality is a challenge and requires further physics understanding. Doping CNTs could be one of the solutions to avoid the unclear metallic or semiconducting property. A modification of the crystalline nanotube by placing defects or foreign atoms brings a tuneable energy band structure, and then a shift of Fermienergy level could help to enhance the electrical properties of CNTs [5]. It is the focus of this paper to provide deeper understanding on doping effects on CNT interconnects. We investigate the physical behaviour and properties of doped CNTs. We perform modelling and simulation and quantify the impact on electrical resistance change for N-type doped CNT interconnects.

The rest of this paper is organized as follows. Section II covers the physical description of doping effect in order to motivate the reason of considering doped CNTs. Section III will show our physical compact model and simulation results for different CNT dimensions. Section IV concludes this paper.

\section{PHYSICAL DESCRIPTION OF DOPING}

\section{A. Hybridization}

In order to understand the fundamental physics about the carbon based 1D structure, hybridization needs to be introduced. It is a chemical concept describing a mix of atomic orbitals with shared electrons under different energies and shapes to form chemical bonds. Electron transport in pure 
CNTs could be understood by learning how CNT lattice hybridizes its internal electrons. Electrical property of doping CNT can also be explained physically by a modification of hybridization.

Hybridization plays a big role in the heteroatom structures. Adding external different atoms will change the lattice strength and surface roughness of the doping material. Hybridization is a chemical description for bonding two atoms with effective electron pairs shared between them. Electrons can be localized or delocalized due to different hybridizations. Doping with a donor atom could increase delocalised electrons in the system in order to give more charge carriers for current transport. Also an acceptor atom doped in the system will create a localised vacuum site enhancing the electron mobility. In a carbon nanotube, each atom has 4 valence electrons, 3 of these electrons participate in the C-C sigma bonding but 1 of them occupies a $P_{z}$ orbital. The $P_{z}$ states mix together forming delocalized electron states with a range of energies that include the Fermi-energy, these states are responsible for the electrical conductivity of graphene or carbon nanotube. One single layer carbon nanotube behaves a $s p^{2}$ hybridization as illustrated below in Figure 1.

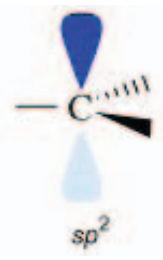

Figure 1. $s p^{2}$ hybridization, 3 in-plane electrons and 1 vertical electrons.

\section{B. Electronic density of state}

Theoretical understanding [6] shows that the conduction in carbon nanotube occurs via the delocalized $\pi$-electron system. At room temperature, both metallic and semiconducting undoped single walled carbon nanotube (SWCNT) are ballistic conductors with two and one spin-degenerate conducting channel(s), respectively. The channels belong to the first $\pi$ - and $\pi^{*}$ - band of the delocalized $\pi$-electron system. In order to have more transport channels, which means involve higher energy bands beyond the first $\pi$-bands, requires a huge electron excitation energy in the order of $1 \mathrm{eV}$, equivalent to the temperature about $1200 \mathrm{~K}$, which is not feasible for circuit interconnect applications. Thus, tuning the energy band gap becomes the key point that can be modified by doping external atoms in order to create extrinsic electron states.

The substitution of carbon atoms in the honeycomb lattice of CNT by other atoms with a different number of valence electrons will in general introduce additional electron states in the density of states (DOS) for CNT. Most general doping atoms are nitrogen and boron which neighboring to carbon atom from the periodical table with one more and less valence electron, and create $\mathrm{N}$ - and $\mathrm{P}$ - type doping respectively. With a graphite-like incorporated nitrogen in CNT, the nitrogen states are found above the Fermi-energy level [7]. As the nitrogen states ionizes, the liberated electrons will occupy empty states at the edge of the valence band and an N-type semiconducting CNT will be created. The Fermi-energy level of the nanotube will shift (relatively) upwards in energy and position itself at the nitrogen states in the DOS. Inversely for the boron doping, due to the one less valence electron, a graphite-like boron (acceptor) can act as electron traps for delocalized $\pi$-electron. If these traps are occupied, that is, the boron states are ionized, the Fermi-energy level will shift downwards in energy towards valance band (see Figure 2). Those two processes can reduce the semiconducting CNT energy gap and enhance the metallic effect.
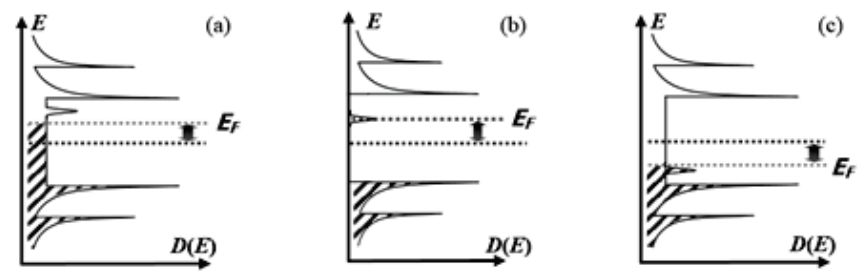

Figure 2. Schematic DOS D(E) as a function of energy $E$ of a metallic SWNT doped by substitution of carbon by (a) nitrogen and (c) by boron in a graphite-like way. The dotted lines represent the initial (dark grey) and the final (grey) position of the Fermi-energy $\mathrm{E}_{\mathrm{F}}$ after ionization of the dopant states. The shift is indicated by an arrow. Hatched regions correspond to occupied states in the DOS. (b) Case of a semiconducting SWNT doped with nitrogen in a graphite-like way [7].

\section{MODELING AND SIMULATIONS}

From electrical point of view, CNT are modelled as an $R L C$ circuit (see Figure 3), which allows to devise analytical formulas to describe the CNT as an interconnect [8, 12-13]. Here, we concentrate on the resistance variance for different CNT dimensions.

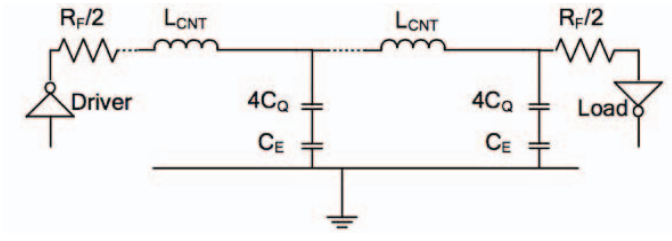

Figure 3. Schematic of electrical RCL model for SWCNT [8].

\section{A. Single walled carbon nanotube}

In this part, we discuss the physical resistance model and simulations about undoped and N-doped single walled CNTs. The resistance of an isolated SWCNT includes intrinsic resistance and scattering resistance, which are due to the quantum transport and electron-phonon scattering, respectively [8].

CNTs could be considered as a quantum wire transport where the conductance is evaluated using the two-terminal LandauerButtiker formula. This states that, a 1-D system with $\mathrm{N}$ 
channels in parallel, the conductance is as $G=\left(N e^{2} / h\right) T$, where $\mathrm{T}$ is the transmission coefficient for electrons pass through the CNT. Due to electron spin degeneracy and two effective atoms per unit cell in graphene, each CNT has four conducting channels in parallel $(\mathrm{N}=4)$, ballistic transport in SWCNT assumes perfect contacts $(\mathrm{T}=1)$, the intrinsic $\mathrm{CNT}$ conductance is given by $4 e^{2} / h$ which yields a resistance about $6.45 \mathrm{~K} \Omega$. For the length of CNT longer than the mean free path (here we take $\mathrm{L}_{\mathrm{MFP}}=1 \mu \mathrm{m}$ ), scattering resistance becomes important and need to be taken into account. Equation (1) shows the total SWCNT resistance as:

$$
R_{S W C N T}=\frac{h}{2 N e^{2}}\left(1+\frac{L}{L_{M F P}}\right)+R_{C}
$$

where $R c$ is the contact resistance for interconnect. Also from the tight-banding calculation, mean free length of electrons in metallic CNT is given as:

$$
L_{M F P}=\frac{D \sqrt{3} \psi^{2}}{2 \sigma_{\varepsilon}^{2}+9 \sigma_{\psi}^{2}}
$$

where there are three tight-binding calculation parameters. For undoped CNT, we chose $\sigma_{\varepsilon}=0.04 \mathrm{eV}$ and $\sigma_{\psi}=0$; for doping $\mathrm{CNT}, \sigma_{\varepsilon}=0$ and $\sigma_{\psi}=0.06 \mathrm{eV}$ [9].

In this model, we analyse a standalone pure CNT and neglect the contact resistance $(\mathrm{Rc}=0)$, which can be introduced by fabrication process. From equation (1) and (2), the resistance of an isolated SWCNT varies proportionally with its length and inversely proportional to its diameter. To perform simulations based on the analytical formulas, we developed Verilog-A models in Cadence environment. These models were used to build different schematic test cases for CNT interconnects as $R L C$ type circuits. Figure 4 illustrates resistance variation due to diameter (D) and length (L) for a pure SWCNT. A really sharp decrease of resistance can be observed by increasing the diameter from 1 to $20 \mathrm{~nm}$ for a long length $(\mathrm{L}=5 \mu \mathrm{m})$. While there are less length impacts on resistance for greater diameter (i.e., D $>5 \mathrm{~nm}$ ). For interconnect design, larger diameter should be considered as priority, but even for a big diameter, $\mathrm{D}=20 \mathrm{~nm}$, resistance of pure SWCNT varies from $6.6 \mathrm{k} \Omega$ to $8.2 \mathrm{k} \Omega$ with increasing of length, which does not have much advantages than conventional copper interconnects. Making SWCNT behaves more conductive, so called more metallic like, doping has been proposed as a solution.

As described earlier, doping can fine tune the Fermi-energy level and add more conducting channels. From the Fermi-Dirac distribution, the number of conducting channels per shell is given by:

$$
N_{\text {channel } / \text { shell }}=\sum_{\text {all subband }} \frac{1}{\exp \left(\left|E_{v}\right| / k_{B} T\right)+1}
$$

where $E_{v}=E_{i}-E_{F}$, the difference between the possible participating electron energy subband and the Fermi-energy level. The value of $E_{v}$ is expected to change when the CNT is doped. In our simulations, in order to investigate the doping impact on CNT resistance, two values of $E_{v}$ have been chosen $\left(E_{v}=0 \mathrm{eV}\right.$ and $\left.E_{v}=1 \mathrm{eV}\right)$, because of the energy bandgap of pure semiconducting SWCNT varies from 0 to $2 \mathrm{eV}$. Since we do not know exactly the bandgap of one SWCNT, an average value $E_{v}=1 \mathrm{eV}$ is chosen to represent a light doping and a heavy doping is represented by $E_{v}=0 \mathrm{eV}$, which makes a doped semiconducting SWCNT behaves just like a metallic SWCNT.

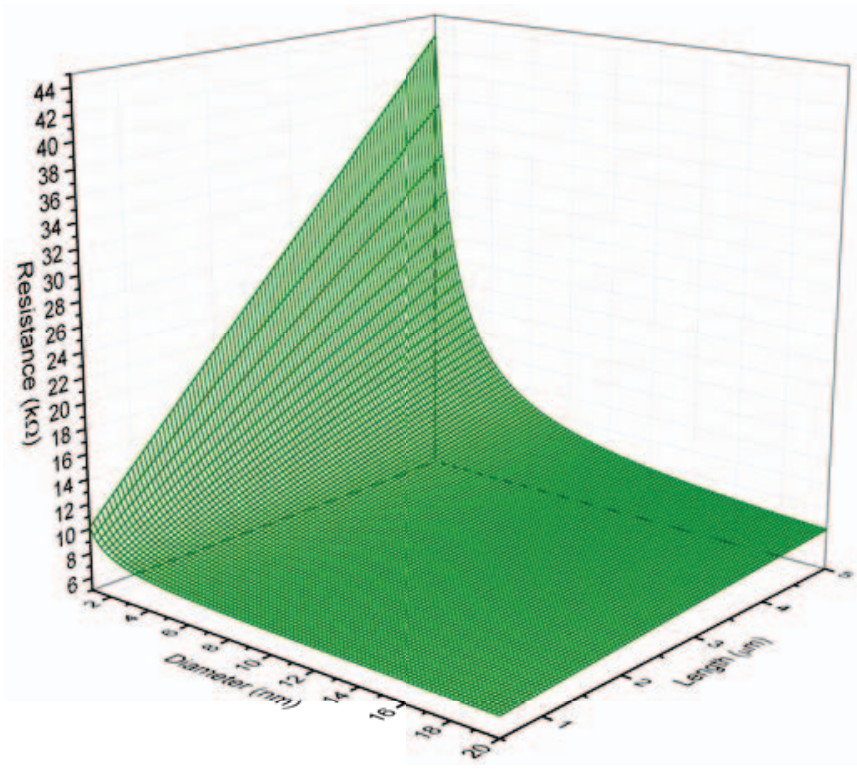

Figure 4. Resistance of pure SWCNT varies with its diameter and length (diameter varying from $1 \mathrm{~nm}$ to $20 \mathrm{~nm}$; length varying from $0.5 \mu \mathrm{m}$ to $5 \mu \mathrm{m})$.

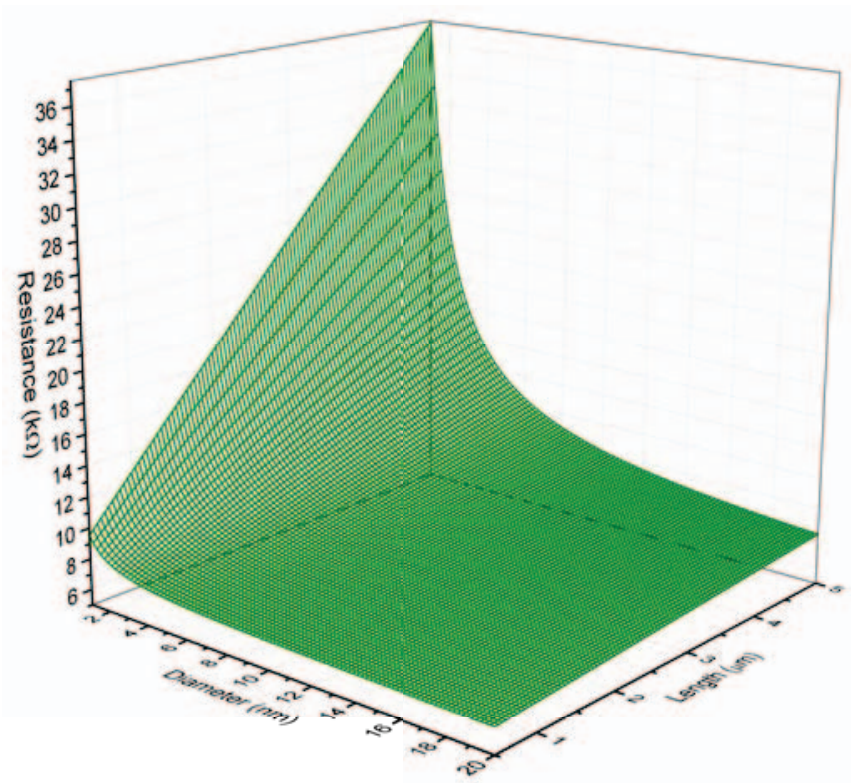

Figure 5. Doped SWCNT resistance varies with its diameter and length for $E_{v}=1 \mathrm{eV}$. 


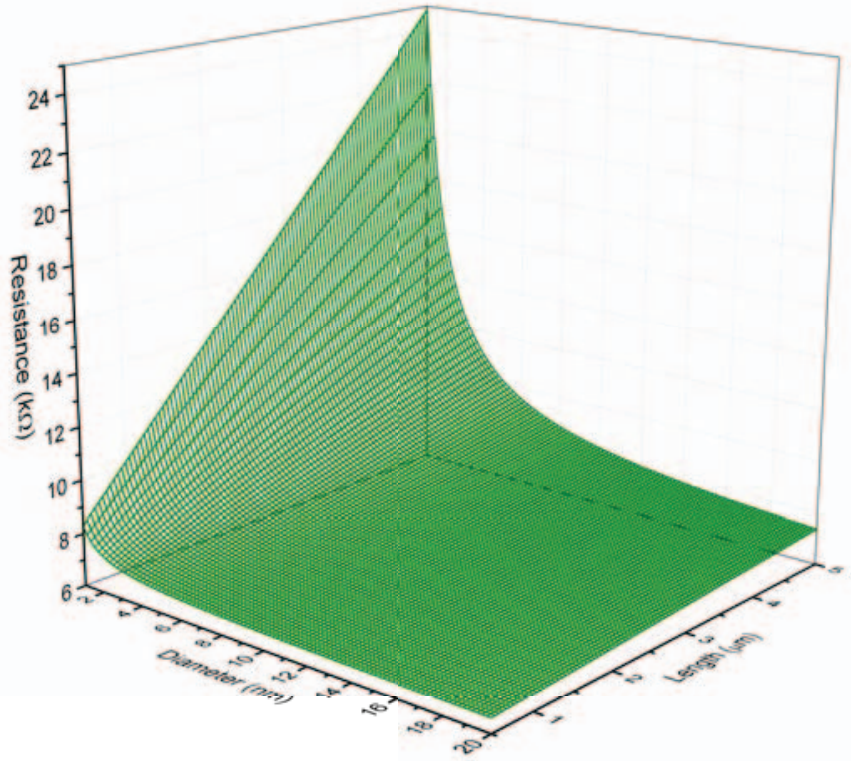

Figure 6. Doped SWCNT resistance varies with its diameter and length for $E_{v}=0 \mathrm{eV}$.

Figures 5 and 6 show the doped SWCNT with $E_{v}=1 \mathrm{eV}$ and $E_{v}=0 \mathrm{eV}$, respectively. If we look at the starting point $(\mathrm{D}=1 \mathrm{~nm}$ and $\mathrm{L}=0.5 \mu \mathrm{m}$ ), resistance decreases from $9.5 \mathrm{k} \Omega$ to $8.3 \mathrm{k} \Omega$ when $E_{v}$ changes from $1 \mathrm{eV}$ to $0 \mathrm{eV}$. Also, for larger diameters and lengths $(\mathrm{D}=5 \mathrm{~nm}$ and $\mathrm{L}=5 \mu \mathrm{m})$, we observe that resistance decreases from $8.3 \mathrm{k} \Omega$ to $7.3 \mathrm{k} \Omega$. And comparing Figure 5 and 6 to Figure 4, with same variations ( $\mathrm{L}$ and $\mathrm{D}$ ), the overall resistance axis scale reduced from $44 \mathrm{k} \Omega$ to $36 \mathrm{k} \Omega$ and $24 \mathrm{k} \Omega$ respectively. This demonstrates that doping do decrease SWCNT resistance.

If we compare the pure CNTs with doped CNTs (see Figure 7), supposing the SWCNT is an idea ballistic transport with a typical mean free path $(\mathrm{L}=1 \mu \mathrm{m})$ and a diameter $\mathrm{D}=1 \mathrm{~nm}$, after light doping (i.e. $\mathrm{E}_{\mathrm{v}}=1 \mathrm{eV}$, energy bandgap of $\mathrm{CNT}$ goes to 1 $\mathrm{eV}$ ) the resistance decrease about $25 \%$ (see Figure 7 (a)). However, with the increase of diameter, doping seems to be less effective, which can be explained by equation (1) and (2), that is, the resistance of SWCNT depends (inversely proportional) on its diameter and the mean free path (i.e., equation (1)). Thus the mean free path is proportional to its diameter with some constants (tight-binding parameters, see equation (2)), then the SWCNT's resistance depends on the diameter and number of conducting channels for any fixed length $\mathrm{L}$. When the diameter becomes greater, which will dominate the value of mean free path, then dominate the resistance and comparable to the behaviour of number of conducting channels. We can say that besides doping, larger diameter also plays an important role for a higher conductivity. For interconnect progress, this observation is important on deciding the CNT interconnect dimensions that leads lower resistance.

From Figure 7 (b), for $\mathrm{D}=1 \mathrm{~nm}$ and $\mathrm{L}=5 \mu \mathrm{m}$, resistance reduced around $40 \%$ after doping, and $11 \%$ of resistance reduction is observed with $\mathrm{D}=20 \mathrm{~nm}$ and $\mathrm{L}=5 \mu \mathrm{m}$ (precise values can be found in Table 1). Comparing to Figure 7 (a) (light doping), the overall resistance is smaller here in heavy doping. However, doping impact is still dominated by diameter but gets more effective. There is a competition between doping and diameter, but both of them will lower SWCNT's resistance. Having a larger diameter and more doping effect could be a better choice for SWCNT interconnect design.

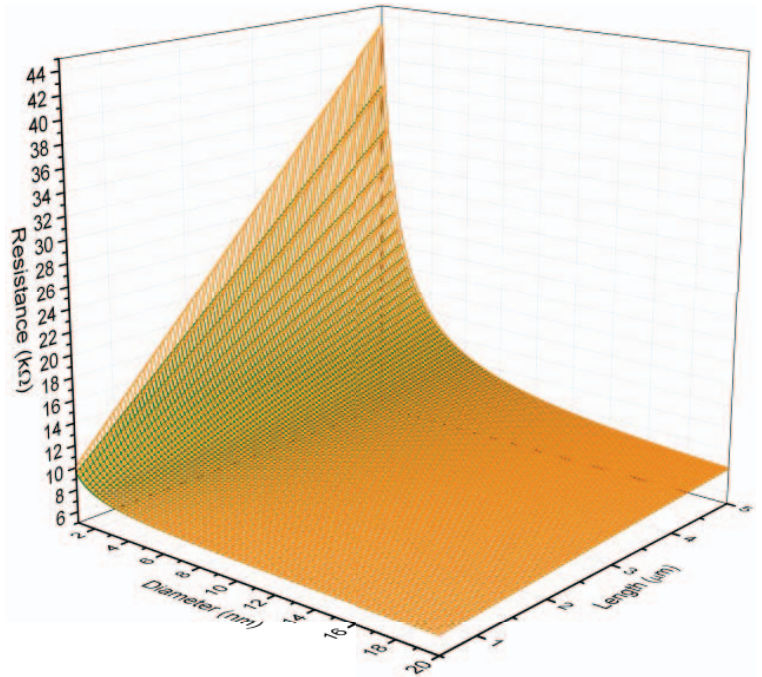

(a)

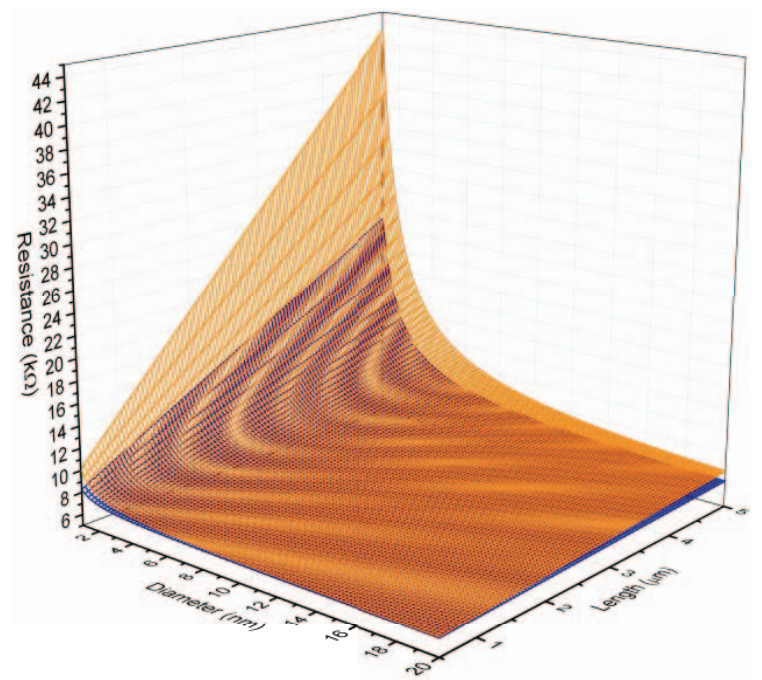

(b)

Figure 7. 3D resistance plots comparison between pure SWCNT and doped SWCNT. (a) shows the pure SWCNT (orange plot) vs light doped SWCNT $\left(\mathrm{E}_{\mathrm{v}}=1 \mathrm{eV}\right.$, green plot). (b) shows the pure SWCNT (orange plot) vs. heavy doped SWCNT $\left(E_{\mathrm{v}}=0 \mathrm{eV}\right.$, purple plot $)$. 
Table 1. Resistance comparison of pure SWCNT with, light doped $\left(E_{\mathrm{v}}=1 \mathrm{eV}\right)$ and heavy doped $\left(\mathrm{E}_{\mathrm{v}}=0 \mathrm{eV}\right)$ SWCNTs.

\begin{tabular}{|c|c|c|c|c|}
\hline \multirow{2}{*}{$\begin{array}{c}\text { Diameter } \\
(\mathrm{nm})\end{array}$} & \multirow{2}{*}{$\begin{array}{c}\text { Length } \\
(\mu \mathrm{m})\end{array}$} & \multicolumn{3}{|c|}{ Resistance (k $\Omega$ ) } \\
\hline & & Pure & $\begin{array}{c}\text { Doped } \\
(E v=1 \text { eV })\end{array}$ & $\begin{array}{c}\text { Doped } \\
(E v=0 \text { eV })\end{array}$ \\
\hline \multirow{3}{*}{1} & 0.5 & 10.01 & 9.55 & 8.29 \\
\hline & 1 & 13.64 & 12.36 & 10.20 \\
\hline & 5 & 43.25 & 37.38 & 24.85 \\
\hline \multirow{3}{*}{5} & 0.5 & 7.12 & 7.09 & 6.78 \\
\hline & 1 & 8.44 & 7.76 & 7.10 \\
\hline & 5 & 13.07 & 12.85 & 9.76 \\
\hline \multirow{3}{*}{10} & 0.5 & 6.81 & 6.80 & 6.63 \\
\hline & 1 & 7.52 & 7.13 & 6.80 \\
\hline & 5 & 10.02 & 9.96 & 8.24 \\
\hline \multirow{3}{*}{15} & 0.5 & 6.70 & 6.69 & 6.58 \\
\hline & 1 & 7.20 & 6.92 & 6.69 \\
\hline & 5 & 8.93 & 8.87 & 7.67 \\
\hline \multirow{3}{*}{20} & 0.5 & 6.64 & 6.64 & 6.55 \\
\hline & 1 & 7.01 & 6.81 & 6.63 \\
\hline & 5 & 8.31 & 8.29 & 7.38 \\
\hline
\end{tabular}

\section{B. Multiwalled carbon nanotube}

The RLC model used in this work (Figure 8) for multi walled carbon nanotube (MWCNT) was introduced in [10].

Resistors are considered in parallel between shells, also the inter-shell tunnelling conductance is considered in the resistance formula (see equation (4)). As known from SWCNT, all conducting channels are in parallel, so the multi walled CNT will have much more parallel conducting channels, thus we can expect to have a smaller resistance. The conductance of MWCNT is expressed as:

$$
G=\frac{2 N e^{2}}{h}\left(\sum_{i} \frac{\beta D_{i}}{\beta D_{i}+L}\right)+\sum_{i}^{N_{S}-1} \sigma \pi D_{i} L
$$

where $\beta$ is the inverse of the mean free path $\mathrm{L}_{\mathrm{MFP}}, \mathrm{N}$ is the number of conducting channels per shell in MWCNT, the diameter of each shell $D_{i}, N_{s}$ is the number of shells, and the $\sigma$ is introduced as a normalized tunnelling conductivity, which the value is $0.3\left(\mu \Omega \cdot \mathrm{cm}^{2}\right)^{-1}$ derived experimentally by [10]. In our simulations, we vary the number of shells from 1 to 20 . Simulations are shown in Figure 9. We observe that the resistance decreases with longer MWCNT lengths and large number of shells. This also indicates that MWCNTs could be better (less resistance) for longer (global) interconnects than SWCNT.

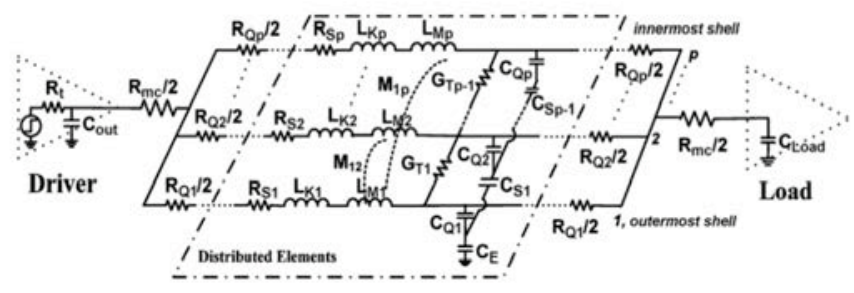

Figure 8. MWCNT electrical RCL model [10].
We also investigate how N-type doping can enhance the conductance for MWCNT interconnects. We simulate the resistance with doping modifications. Since we know from the theoretical understanding, that doping will open more conducting channels for electrons, we investigate how the number of conducting channels impact the resistance. Here, we chose two different shell number, $\mathrm{N}_{\mathrm{S}}=1$ and $\mathrm{N}_{\mathrm{S}}=5$, and vary the number of conducting channels per shell, because that, as mentioned before, the diameter will impact the resistance, but, here we are more interested in the doping effects. With a larger number of shells, which means that the MWCNT has a larger outer diameter (i.e. distance between shells is fixed for $0.34 \mathrm{~nm}$ corresponding to the lattice length of carbon-carbon bonding length), we obtain that the resistance of MWCNTs is always smaller. Simulations are shown in Figure 10 and 11. Increasing the number of conducting channels per shell can lead to a significant decrease in resistance. These simulations clearly demonstrate again that doping can really help for improving electrical conductivity for both SWCNT and MWCNT.

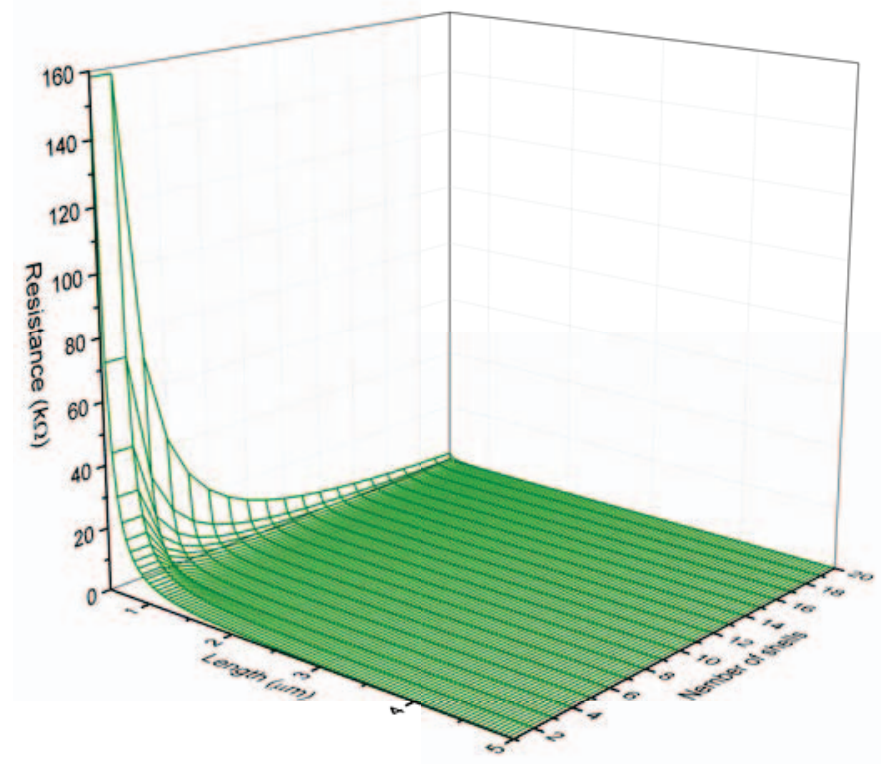

Figure 9. Resistance of a pure MWCNT for various lengths and number of shells.

Looking at Figure 12, one-shell $\left(\mathrm{N}_{\mathrm{S}}=1\right)$ MWCNT has larger variation in resistance than five-shell $\left(\mathrm{N}_{\mathrm{S}}=5\right)$ MWCNT. For $\mathrm{L}=1 \mu \mathrm{m}$, ideal ballistic transport length, resistance decrease around $89 \%$ with varying number of conducting channels from 2 to 10 per shell for both 1-shell and 5-shells CNT. An important observation is that with a long length $(\mathrm{L}=5 \mu \mathrm{m})$ and 10 conducting channels per shell, a resistance of about $1.8 \mathrm{k} \Omega$ can be obtained for 1-shell CNT, and for 5-shell CNT, a very low resistance about $0.3 \mathrm{k} \Omega$ is observed, which is very impressive for a CNT interconnect for on-chip application and give a promising future to replace the conventional copper interconnect. Again, the doping impact has been demonstrated. This tells that N-type doped MWCNT could be a very good global interconnect. How to increase the number of conducting channels per shell is part of on-going and future research on enhancing doping effect on CNTs, which ultimately can pave the way on how to exploit CNTs for on-chip interconnects. 


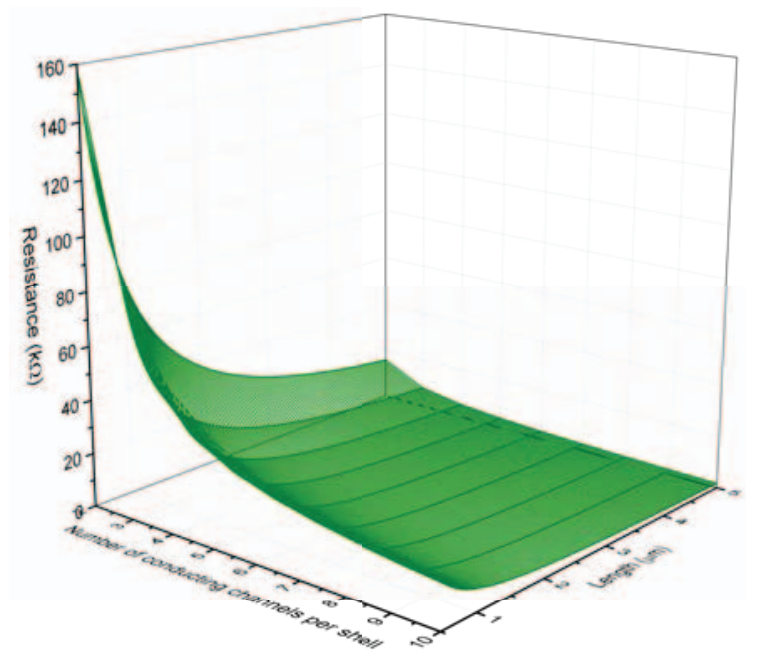

Figure 10. Resistance of MWCNT with $\mathrm{N}_{\mathrm{S}}=1$ for different lengths and conducting channels per shell.

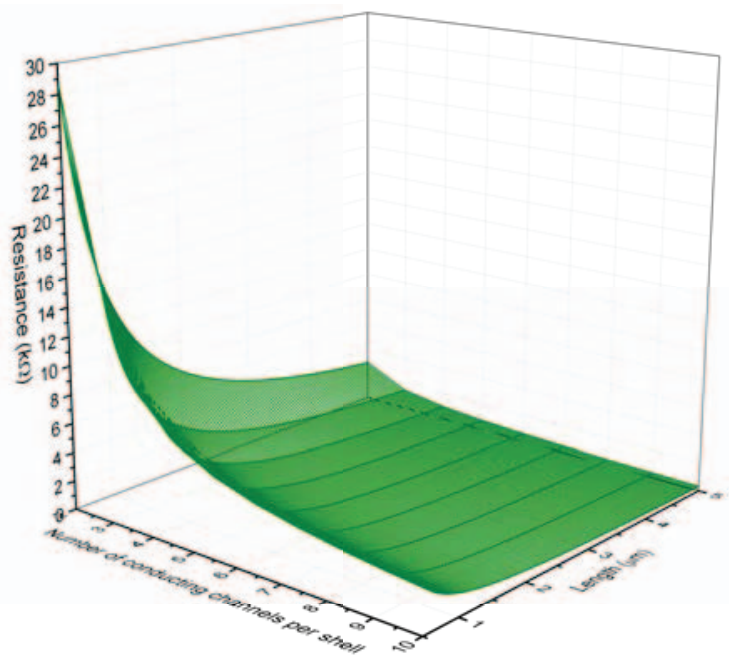

Figure 11. Resistance of MWCNT with $\mathrm{N}_{\mathrm{S}}=5$ for different lengths and conducting channels per shell.

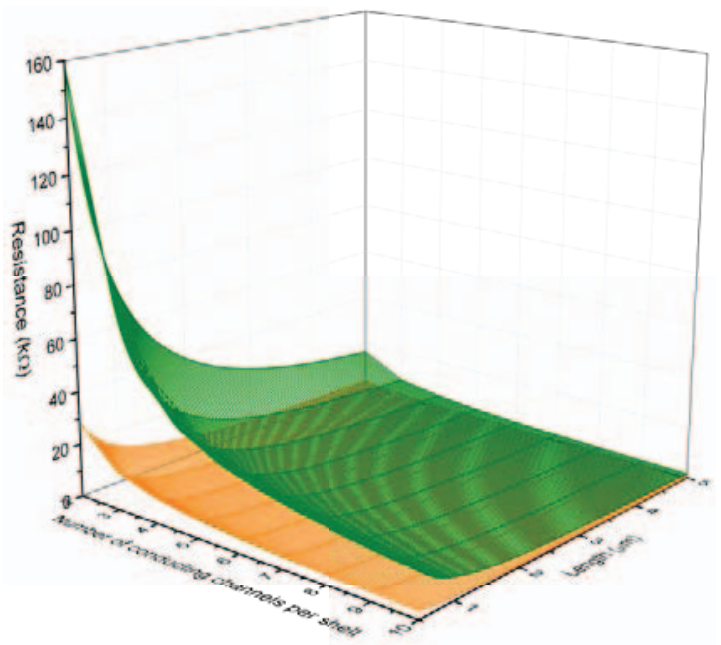

Figure 12. Resistance plots comparison between one-shell (green plot) and five-shell (orange plot) MWCNT.

\section{CONCLUSION}

In this paper, we investigate doped carbon nanotubes as potential on-chip interconnect material. We also provide physical description on pure and doping impact on CNT electrical properties. Our simulations on different CNT dimensions show a decrease in resistance while increasing diameter and number of conducting channels (i.e., increasing doping effect) for CNTs. We obtain for SWCNT a reduction of about $11 \%$ of resistance for a larger diameter $(D=20 \mathrm{~nm})$ and longer length $(\mathrm{L}=5 \mu \mathrm{m})$ after heavy doped. Moreover, MWCNTs show greater promise as doping can reduce resistance significantly up to $89 \%$ by varying the conducting channels per shell from 2 to 10 . Additionally, an impressive observation with a $0.3 \mathrm{k} \Omega$ resistance for 5 -shell $\mathrm{CNT}$ with 10 conducting channels per shell shows a hopeful future for replacing conventional copper interconnect. This work incites more physical investigation on doping CNT research to improve the CNT performances as interconnect applications for large-scale integrated circuit.

\section{ACKNOWLEDGMENT}

Authors would like to thank H2020 CONNECT European project. This project has received funding from the European Union's Horizon 2020 research and innovation programme under grant agreement No 688612 .

\section{REFERENCES}

[1] P. R. Wallace, "The band structure of graphite," Physical Review, vol. 71, no. 9, pp. 622-634, 1947

[2] S. Iijima, "Helical microtubules of graphitic carbon," Nature, vol. 354(6348), pp. 56-58, 1991.

[3] A. K. Geim, "Graphene: status and prospects," Science, vol. 324(5934), pp. 1530-1534, 2009.

[4] A. K. Geim, K. S. Novoselov, "The rise of graphene," Nature Materials, vol. 6, no. 3, pp. 183-191, 2007.

[5] P. Ayala, R. Arenal, M. Rummeli, A. Rubio, T. Picher, "The doping of carbon nanotubes with nitrogen and their potential applications," Carbon, vol. 48, pp. 575-586, 2010.

[6] M. Glerup, V. Krstić, C. Ewels, M. Holzinger, G. Van Lier, "Doping of carbon nanotubes," in Doped Nanomaterials and Nanodevices, Edited by Wei Chen. American Scientific Publishers 2007.

[7] F. V. Paez, "Effects of Doping Single and Double Walled Carbon Nanotubes with Nitrogen and Boron," Master thesis at MIT 2006.

[8] N. Srivastava and K. Banerjee, "Performance analysis of carbon nanotube interconnects for VLSI applications," IEEE/ACM International Conference on Computer Aided Design, 2005.

[9] J. Jiang, J. Dong, H. T. Yang, and D. Y. Xing, "Universal expression for localization length in metallic carbon nanotubes," Physical Review Letter, vol. 64, no. 4, 2 July 2001.

[10] H. Li, W. Y. Yin, K. Banerjee, and J. F. Mao, "Circuit modeling and performance analysis of multi-walled carbon nanotube interconnects, " IEEE Transactions on Electron Devices, vol. 55, no. 6, June 2008.

[11] B. Burlon, C. Miko, L. Forro, D. C. Glattli, and A. Bachtold, "Determination of the intershell conductance in multiwalled carbon nanotubes, " Physical Review Letter, vol. 93, no. 17, Oct 2004.

[12] A. Todri-Sanial, J. Dijon, A. Maffucci, "Carbon nanotube interconnects: process, design and application," Springer 2016.

[13] A. Todri-Sanial, "Lumped electro-thermal modeling and analysis of carbon nanotube interconnects," in Nano-CMOS and Post-CMOS Electronics: Circuits and Design, Edited by S. P. Mohanty and A. Srivastava, The Institute of Engineering and Technology (IET), 2016. 